\title{
Outcomes of Burkitt lymphoma with central nervous system involvement: evidence from a large multicenter cohort study
}

Haematologica 2021

Volume 106(7):1932-1942

Presented in part as an Oral Presentation at the $61^{\text {st }}$ American Society of Hematology Meeting \& Exposition, December 7-10, 2019, in Orlando, FL, USA.

\section{Correspondence:}

ADAM J. OLSZEWSKI

adam_olszewski@brown.edu

Received: September 6, 2020.

Accepted: December 22, 2020.

Pre-published: February 4, 2021.

https://doi.org/10.3324/haematol.2020.270876

\section{(C2021 Ferrata Storti Foundation}

Material published in Haematologica is covered by copyright. All rights are reserved to the Ferrata Storti Foundation. Use of published material is allowed under the following terms and conditions:

https://creativecommons.org/licenses/by-nc/4.0/legalcode. Copies of published material are allowed for personal or internal use. Sharing published material for non-commercial purposes is subject to the following conditions:

https://creativecommons.org/licenses/by-nc/4.0/legalcode, sect. 3. Reproducing and sharing published material for commercial purposes is not allowed without permission in writing from the publisher.

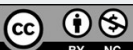

Adam S. Zayac,,$^{1 *}$ Andrew M. Evens,,$^{2 *}$ Alexey Danilov, ${ }^{3}$ Stephen D. Smith, ${ }^{4}$ Deepa Jagadeesh, ${ }^{5}$ Lori A. Leslie, ${ }^{6}$ Catherine Wei, ${ }^{2}$ Seo-Hyun Kim, ${ }^{7}$ Seema Naik, ${ }^{8}$ Suchitra Sundaram, ${ }^{9}$ Nishitha Reddy, ${ }^{10}$ Umar Farooq, ${ }^{11}$ Vaishalee $P$ Kenkre, ${ }^{12}$ Narendranath Epperla, ${ }^{13}$ Kristie A. Blum, ${ }^{14}$ Nadia Khan, ${ }^{15}$ Daulath Singh, ${ }^{16}$ Juan P. Alderuccio, ${ }^{17}$ Amandeep Godara, ${ }^{18}$ Maryam Sarraf Yazdy, ${ }^{19}$ Catherine Diefenbach, ${ }^{20}$ Emma Rabinovich, ${ }^{21}$ Gaurav Varma, ${ }^{22}$ Reem Karmali, ${ }^{23}$ Yusra Shao, ${ }^{5}$ Asaad Trabolsi, ${ }^{17}$ Madelyn Burkart, ${ }^{23}$ Peter Martin, ${ }^{22}$ Sarah Stettner, ${ }^{21}$ Ayushi Chauhan, ${ }^{19}$ Yun Kyong Choi, ${ }^{20}$ Allandria StrakerEdwards, ${ }^{15}$ Andreas Klein, ${ }^{18}$ Michael C. Churnetski, ${ }^{14}$ Kirsten M. Boughan, ${ }^{24}$ Stephanie Berg, ${ }^{16}$ Bradley M Haverkos, ${ }^{25}$ Victor M. Orellana-Noia, ${ }^{26}$ Christopher D'Angelo, ${ }^{12}$ David A Bond, ${ }^{13}$ Seth M. Maliske, ${ }^{11}$ Ryan Vaca, ${ }^{8}$ Gabriella Magarelli, ${ }^{6}$ Amy Sperling, ${ }^{4}$ Max J. Gordon, ${ }^{3}$ Kevin A. David, ${ }^{2}$ Malvi Savani, ${ }^{27}$ Paolo Caimi, ${ }^{24}$ Manali Kamdar, ${ }^{25}$ Matthew A. Lunning, ${ }^{28}$ Neil Palmisiano, ${ }^{29}$ Parameswaran Venugopal, ${ }^{7}$ Craig A Portell, ${ }^{26}$ Veronika Bachanova, ${ }^{27}$ Tycel Phillips, ${ }^{30}$ Izidore S. Lossos ${ }^{17}$ and Adam J. Olszewski ${ }^{1}$

${ }^{1}$ Lifespan Cancer Institute, Alpert Medical School of Brown University, Providence, RI; ${ }^{2}$ Rutgers Cancer Institute of New Jersey, Robert Wood Johnson University Hospital, New Brunswick, NJ; ${ }^{3}$ Knight Cancer Institute, Oregon Health \& Science University, Portland, OR; ${ }^{4}$ University of Washington/Fred Hutchinson Cancer Research Center, Seattle, WA; ${ }^{5}$ Taussig Cancer Institute, Cleveland Clinic, Cleveland, OH; ${ }^{6} J o h n$ Theurer Cancer Center, Hackensack University Medical Center, Hackensack, NJ; ${ }^{7}$ Rush University Medical Center, Chicago, IL; ${ }^{8}$ Penn State Cancer Institute, Penn State University College of Medicine, Hershey, PA; ${ }^{9}$ Roswell Park Comprehensive Cancer Center, Buffalo, NY; ${ }^{10}$ Vanderbilt University Medical Center, Nashville, TN; ${ }^{11}$ University of lowa Carver College of Medicine, lowa City, IA; ${ }^{12}$ University of Wisconsin Carbone Cancer Center, Madison, WI; ${ }^{13}$ The Ohio State University Comprehensive Cancer Center, Columbus, $\mathrm{OH} ;{ }^{14}$ Winship Cancer Institute, Emory University, Atlanta, GA; ${ }^{15} \mathrm{Fox}$ Chase Cancer Center, Philadelphia, PA; ${ }^{16}$ Loyola University Medical Center, Loyola University Chicago, Maywood, IL; ${ }^{17}$ Sylvester Comprehensive Cancer Center, University of Miami School of Medicine, Miami, FL; ${ }^{18}$ Tufts Medical Center, Boston, MA; ${ }^{19}$ Lombardi Comprehensive Cancer Center, Georgetown University Hospital, Washington, DC; ${ }^{20}$ New York University School of Medicine, Perlmutter Cancer Center, New York, NY; ${ }^{21}$ University of Illinois at Chicago, Chicago, IL; ${ }^{22}$ Weill Cornell Medical College, New York, NY; ${ }^{23}$ Northwestern University, Chicago, IL; ${ }^{24}$ University Hospitals Seidman Cancer Center, Cleveland, $\mathrm{OH}$; ${ }^{25}$ University of Colorado Cancer Center, Aurora, CO; ${ }^{26}$ University of Virginia School of Medicine, Charlottesville, VA; ${ }^{27}$ University of Minnesota, Minneapolis, MN; ${ }^{28}$ University of Nebraska Medical Center, Omaha, NE; ${ }^{29}$ Sidney Kimmel Cancer Center, Thomas Jefferson University, Philadelphia, PA and ${ }^{30}$ University of Michigan, Ann Arbor, MI, USA

${ }^{\star} A S Z$ and $A M E$ contributed equally as co-first authors.

\section{ABSTRACT}

entral nervous system (CNS) involvement in Burkitt lymphoma poses a major therapeutic challenge, and the relative ability of contemporary regimens to treat CNS involvement remains uncertain. We describe the prognostic significance of CNS involvement and the incidence of CNS recurrence/progression after contemporary immunochemotherapy using real-world clinicopathological data from adults with Burkitt lymphoma diagnosed between 2009 and 2018 in 30 institutions in the USA. We examined associations between baseline CNS involvement, patients' characteristics, complete response rates, and survival. We also examined risk factors for CNS recurrence. Of 641 patients (aged 18 to 88 years), 120 (19\%) had CNS involvement. CNS involvement was independently associated with human immunodeficiency virus infection, poor performance status, involvement of $\geq 2$ extranodal sites, and bone marrow involvement. Selection of the first-line treatment regimen was unaffected by CNS involvement $(P=0.93)$. Patients with CNS 
disease had significantly lower rates of complete response $(59 \%$ vs. $77 \%$ for patients with and without CNS involvement, respectively; $P<0.001$ ), worse 3 -year progression-free survival (adjusted hazard ratio $[\mathrm{aHR}]=1.53,95 \%$ confidence interval $[95 \% \mathrm{CI}]: 1.14-2.06 ; P=0.004)$ and overall survival $(\mathrm{aHR}=1.62,95 \%$ CI: $1.18-2.22 ; P=0.003)$. The 3 -year cumulative incidence of CNS recurrence was $6 \%(95 \% \mathrm{CI}: 4-8 \%)$ and was significantly lower among patients receiving other regimens (CODOX-M/IVAC, 4\%, or hyperCVAD/MA, 3\%) compared with DA-EPOCH-R (13\%; adjusted sub-distribution $\mathrm{HR}=4.38,95 \% \mathrm{CI}$ :, 2.16-8.87; $P<0.001)$. Baseline CNS involvement in Burkitt lymphoma is relatively common and portends inferior prognosis independently of the first-line treatment regimen selected. In real-world practice, regimens including intravenous systemic agents with pronounced CNS penetrance were associated with a lower risk of CNS recurrence. This finding may be influenced by observed suboptimal adherence to the strict CNS staging and intrathecal therapy procedures incorporated in the DA-EPOCH-R regimen.

\section{Introduction}

Central nervous system (CNS) involvement is a serious complication of Burkitt lymphoma (BL), with an incidence ranging from $5 \%$ to $40 \%{ }^{1-7}$ Most first-line regimens employ dedicated CNS-directed strategies which typically include intrathecal and systemic chemotherapy capable of penetrating the blood-brain barrier. The need for multiple intrathecal injections and potential severe toxicities of high-dose methotrexate (HDMTX) pose challenges to effective CNSdirected therapy, requiring expertise from the clinician and strict adherence by the patient. It is uncertain whether the application and outcomes of CNS-directed treatments in routine clinical practice correspond to those in clinical trials.

Short-cycle, dose-intensive immunochemotherapy regimens achieve progression-free survival (PFS) rates of 70$80 \%$ in BL. ${ }^{8-11}$ Adverse effects associated with high-intensity regimens have limited the use of such regimens among the elderly or patients positive for human immunodeficiency virus (HIV), leading to interest in less aggressive options. The DA-EPOCH-R regimen (dose-adjusted etoposide, prednisone, vincristine, cyclophosphamide, doxorubicin, and rituximab) has demonstrated excellent survival and relatively low toxicity in single-arm trials, indicating that treatment for BL can be de-escalated without apparent loss of efficacy. ${ }^{12,13}$ Whereas DA-EPOCH-R involves the same or larger numbers of intrathecal chemotherapy administrations as those in common high-intensity regimens, it notably lacks classical CNS-penetrant systemic agents (HDMTX, cytarabine, ifosfamide) used for prophylaxis against CNS disease. ${ }^{14}$ DA-EPOCH-R requires strict CNS staging procedures (using flow cytometry of the cerebrospinal fluid [CSF] and brain imaging) and specific protocols for prophylactic or therapeutic intrathecal therapy to control the CNS invasion. Although the National Comprehensive Cancer Network (NCCN) guidelines recommend DA-EPOCH-R as a first-line option for BL along with R-CODOX-M/IVAC (rituximab, cyclophosphamide, doxorubicin, vincristine, and HDMTX, alternating with ifosfamide, etoposide, and cytarabine) and RhyperCVAD/MA (rituximab, cyclophosphamide, vincristine, doxorubicin, and dexamethasone alternating with HDMTX/cytarabine), there are limited data about the efficacy of DA-EPOCH-R in individuals with CNS involvement. The guidelines point out that high-risk patients presenting with symptomatic CNS disease should start treatment with the portion of systemic therapy that contains CNS-penetrating drugs, and that patients with parenchymal brain involvement were not included in the clinical trials of DA-EPOCH-R. ${ }^{15}$
Our objective was to describe factors associated with CNS involvement in $\mathrm{BL}$ using a large, multi-institutional dataset designed to study practice patterns and outcomes of adult BL. ${ }^{16}$ We examined real-world practice of CNSdirected management, and also compared CNS-related outcomes among patients treated with DA-EPOCH-R or with 'high-intensity' regimens.

\section{Methods}

\section{Study cohort}

In this multicenter retrospective study, we included patients aged $\geq 18$ years diagnosed with BL between 2009 and 2018 in 30 institutions throughout the USA. The study was approved by the institutional review boards of all the institutions and waiver of informed consent was accepted. Of 702 identified subjects, 641 had complete clinicopathological data and were entered into a centralized database. Sixty-one patients were excluded because of pathology inconsistent with $\mathrm{BL}(\mathrm{n}=21)$, inadequate follow-up $(n=15)$, treatment dates out of range $(n=13)$, or lack of clinical details $(n=12)$.

The diagnosis was established by local review of pathology reports. BL was defined according to the World Health Organization (WHO) criteria, ${ }^{17}$ excluding other entities such as high-grade B-cell lymphoma, not otherwise specified, or double/triple-hit lymphoma. We included cases without known MYC rearrangement if they fulfilled other criteria for BL: smallcell morphology with tingible body macrophages, BCL2-negative, CD10/BCL6-positive immunophenotype, and Ki67 staining at $\sim 100 \%$. Staging evaluations and therapy were completed at the discretion of treating physicians.

\section{Variables and endpoints}

Investigators collected data using a standardized protocol. Performance status (PS) was assigned according to the Eastern Cooperative Oncology Group (ECOG) scale. CNS involvement was classified as leptomeningeal (based on CSF evaluation, radiological invasion of meninges or cavernous sinus, or clinical cranial nerve palsy) or parenchymal (radiological or biopsyproven invasion of the brain, eye, or spinal cord), and always classified as stage 4 lymphoma. All CNS evaluations were performed according to institutional standards; specific use of imaging, CSF cytology, flow cytometry, or IGH polymerase chain reaction was not recorded. Serum lactate dehydrogenase (LDH) level was standardized relative to institutional upper limit of normal (ULN). Details about the location of CNS recurrence and schedules of intrathecal therapy were collected for a subset of patients who either experienced a CNS recurrence, or who received DA-EPOCH-R with known baseline CNS 
involvement. PFS was assessed locally as the time from diagnosis until disease progression, recurrence, or death. ${ }^{18}$ Overall survival (OS) was calculated from diagnosis until death or last follow-up.

\section{Statistical analysis}

We compared clinicopathological characteristics between groups using Fisher exact tests and evaluated factors associated with baseline CNS involvement by univariate and multivariable logistic regression (reporting adjusted odds ratios, aOR). Associations with survival were examined in proportional hazard models, first univariate, and then stratified by general $\mathrm{BL}$ risk factors identified in the same dataset, reporting hazard ratios (HR). ${ }^{16}$ The cumulative incidence of CNS recurrence was studied in competing-risk models that accounted for other events such as systemic recurrence or death from any cause, reporting sub distribution hazard ratios (SHR). ${ }^{19}$ To address missing data on PS $(7 \%)$, stage $(2 \%)$, HIV positivity $(2 \%), \mathrm{LDH}$ $(7 \%)$, hemoglobin $(5 \%)$, and albumin values $(9 \%)$, we averaged model coefficients and standard errors from 15 datasets using multiple imputation by chained equations. ${ }^{20}$ The imputation model included all covariates and outcomes. Estimates report $95 \%$ confidence intervals (in square brackets), and two-sided $P$ values $<0.05$ were considered statistically significant.

\section{Results}

The study included 641 patients with untreated BL diagnosed at a median age of 47 years (interquartile range [IOR], $34-59$ years), who were predominantly male $(76 \%)$ and had stage 4 disease $(73 \%$ ) (Table 1). The most common first-line regimens were CODOX-M/IVAC (30\%), hyperCVAD/MA $(30 \%)$, or DA-EPOCH-R (28\%), and $90 \%$ of all patients received rituximab. Eight patients (1\%) did not receive any chemotherapy. Intrathecal chemotherapy was given to 545 patients (85\%) whereas $396(62 \%)$ received systemic HDMTX as part of their first-line treatment regimen. The median follow-up was 45 months.

\section{Baseline central nervous system involvement}

CNS involvement was present at diagnosis in 120 patients (19\%), including 97 (15\%) with leptomeningealonly disease, $20(3 \%)$ with parenchymal disease (of whom 11 had concurrent leptomeningeal disease), and three $(1 \%)$ with unspecified CNS involvement (Figure 1A). CSF was positive in 91 patients ( $14 \%$ of all cases, and $76 \%$ of those with CNS involvement), whereas ten patients had cavernous sinus involvement. Parenchymal disease included brain, ocular, and spinal cord invasion in

Table 1. Patient characteristics stratified by central nervous system involvement at diagnosis.

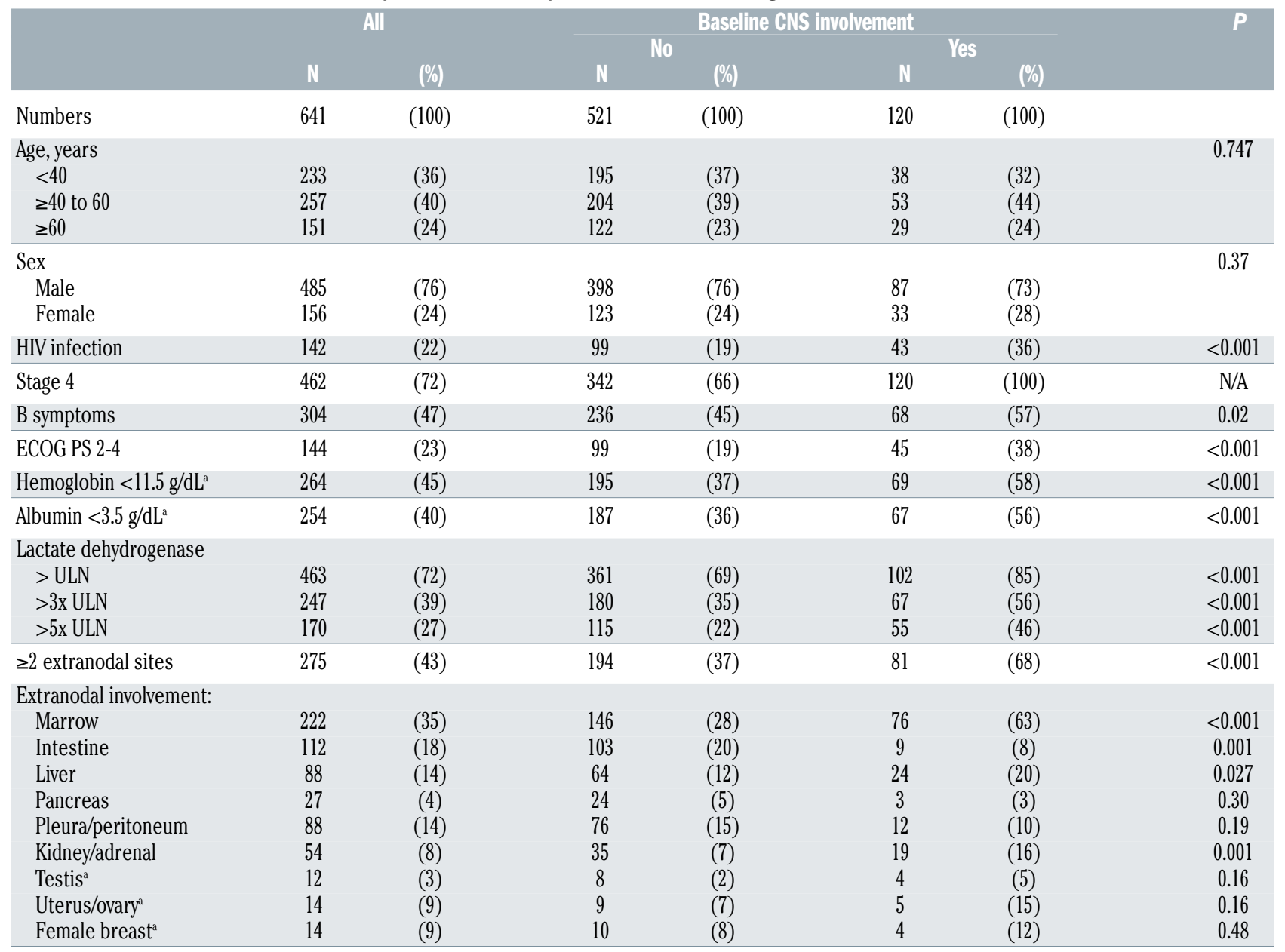

${ }^{a}$ Cutoffs were empirically determined to provide optimal prognostic discrimination in the main study based on this dataset. ${ }^{16}$ bercentages and $P$ values calculated for men or women only, as pertinent. CNS: central nervous system;ECOG PS: Eastern Cooperative Oncology Group performance status; HIV: human immunodeficiency virus; N/A: not applicable; ULN: upper limit of normal. 
nine, seven, and four patients, respectively.

The patients' characteristics associated with baseline CNS involvement in univariate analysis included HIV infection, B symptoms, PS 2-4, low hemoglobin or albumin, high $\mathrm{LDH}, \geq 2$ non-CNS extranodal sites of involvement, and invasion of the bone marrow, liver, kidneys, or adrenal glands (Online Supplementary Table S1). Conversely, patients with extranodal involvement of the intestine were less likely to present with CNS disease. In a multivariate model, HIV infection (aOR: 1.84 [1.12-3.03], $P=0.017$ ), poor PS $(\mathrm{aOR}=2.13 \quad[1.27-3.57], P=0.004), \geq 2$ extranodal sites $(\mathrm{aOR}=2.94 \quad[1.75-4.94], P<0.001)$, and bone marrow involvement $(\mathrm{aOR}=2.80$ [1.59-4.94], $P<0.001)$ retained statistical significance, whereas intestinal involvement was consistently associated with a lower risk $(\mathrm{aOR}=0.34[0.16$ 0.72], $P=0.005$ )

The use of first-line regimens did not differ significantly according to baseline CNS involvement $(P=0.93)$ (Figure 1B). Furthermore, we observed no significant difference in receipt of any intrathecal chemotherapy (89\% vs. $84 \%$, respectively; $P=0.16$ ) or systemic HDMTX (67\% vs. $61 \%$, respectively; $P=0.22$ ). Radiation therapy was used somewhat more frequently in patients with CNS disease $(11 \%$ vs. $6 \%, P=0.05)$, but data on the specific radiation target were not available. Among 20 patients with parenchymal CNS involvement, four (20\%) received DA-EPOCH-R, seven $(35 \%)$ received CODOX-M/IVAC, eight (40\%) received hyperCVAD/M, and one received a low-intensity regimen.

Among $35 \mathrm{BL}$ patients given DA-EPOCH-R who had CNS invasion, 29 (83\%) had only leptomeningeal disease, four $(11 \%)$ had parenchymal disease, and two (6\%) had unspecified involvement. Thirty-four of these 35 patients received care in academic centers. Details of intrathecal administrations were available for 21 patients (60\%). Although all 21 received intrathecal chemotherapy, only $45 \%$ followed the strict schedule from the original protocol (first twice-weekly, then weekly, and then monthly administration). The median number of intrathecal methotrexate administrations was eight (IOR, 5-12). Clearance of CSF disease was recorded in $89 \%$ of patients.

\section{Outcomes of patients with baseline central nervous system involvement}

Patients with baseline CNS involvement had a significantly lower probability of attaining a complete response:
A

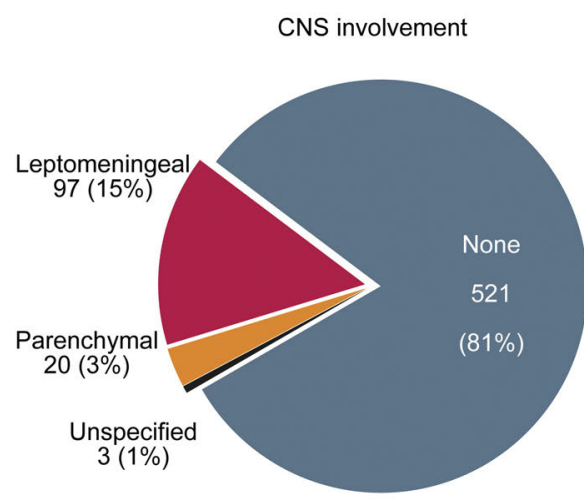

C

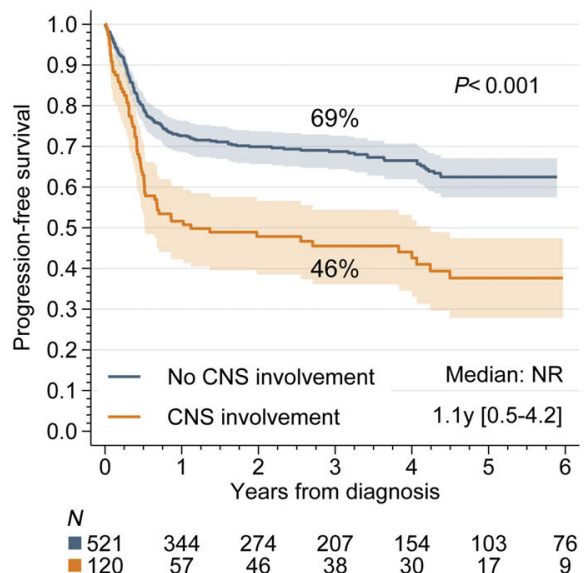

B
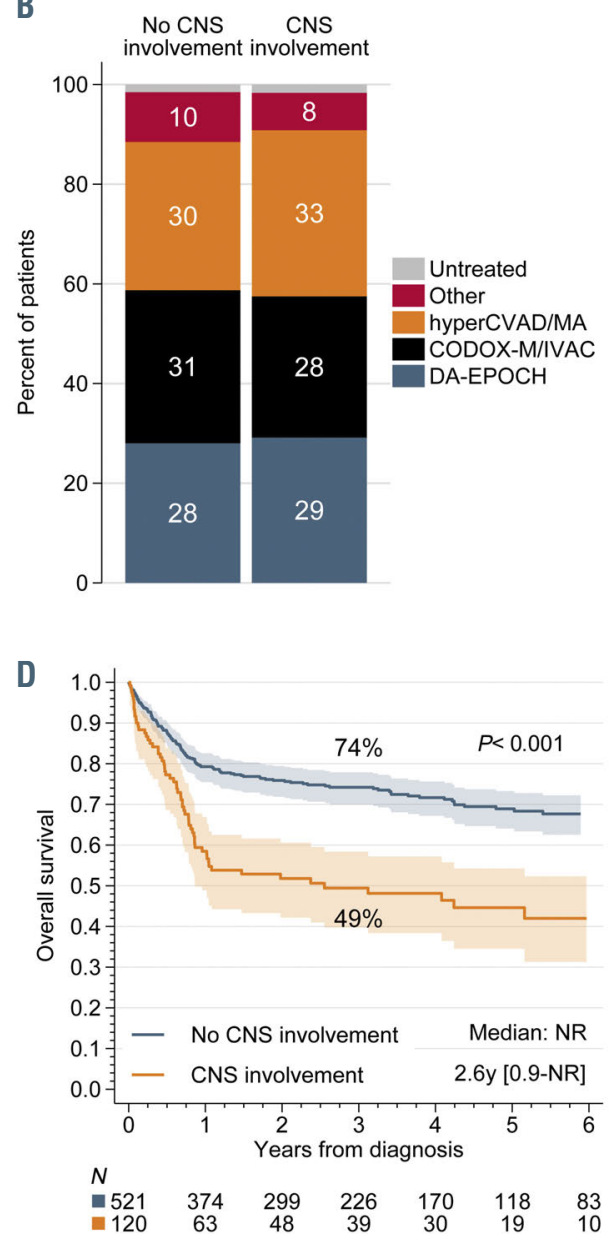

Figure 1. Baseline central nervous system involvement in Burkitt lymphoma. (A) Proportions of patients with leptomeningeal, parenchymal, or unspecified centra nervous system (CNS) involvement; 11 patients with concurrent leptomeningeal and parenchymal disease are included in the last group. (B) Use of first-line chemotherapy regimens stratified by the presence of baseline CNS involvement. (C) Progression-free survival stratified by the presence of CNS involvement. (D) Overall survival stratified by the presence of CNS involvement. Shaded areas indicate $95 \%$ confidence interval bands; 3-year survival estimates and $P$-values from log-rank test are listed. NR: not reached. 
$59 \%$ versus $77 \%$ in patients with or without CNS disease, respectively $(\mathrm{OR}=0.45$ [0.29-0.69], $P<0.001$; excluding untreated patients) as well as worse PFS (3-year estimate, $46 \%$ vs. 69\%, respectively; $\mathrm{HR}=2.02$ [1.52-2.67], $P<0.001)$ (Figure 1C) and OS (49\% vs. 74\%, respectively; HR=2.18 [1.61-2.94], $P<0.001$ ) (Figure 1D). Patients with CNS involvement had a median PFS of 1.1 years [range, 0.5-4.2] and OS of 2.6 years [0.9 to not reached], whereas medians for those without CNS disease were not reached.

CNS involvement remained an independent risk factor for PFS (adjusted $\mathrm{HR}=1.53$ [1.14-2.06], $P=0.004$ ) and $\mathrm{OS}$ (adjusted $\mathrm{HR}=1.62$ [1.18-2.22], $P=0.003$ ) after adjustment for other characteristics independently associated with poor outcomes: age $\geq 40$ years, ECOG PS $2-4$, and LDH level $>3 x U L N .{ }^{16}$ We observed no difference in PFS based on whether the CNS involvement was parenchymal or leptomeningeal only (log-rank $P=0.90$ ), but patients with CNS disease who were diagnosed at age $\geq 60$ years had worse survival (Online Supplementary Figure S1A-D). As expected, the four patients with parenchymal CNS disease who received DA-EPOCH-R had particularly poor outcomes (3year PFS $25 \%$ vs. $57 \%$ for those treated with CODOXM/IVAC and $56 \%$ for those given hyperCVAD/MA) (Online Supplementary Figure S1E and F).

Inferior PFS with baseline CNS involvement was observed regardless of the first-line treatment regimen given $\left(P_{\text {weanation }}=0.85\right)$ (Figure 2A-C) or whether rituximab was used $\left(P_{\text {manasoun }}=0.75\right)$. Similarly, complete response rates were lower with baseline CNS involvement across regimens $\left(P_{\text {menasion }}=0.95\right)$ (Figure 2D) and independent of rituximab use $\left(P_{\text {interacion }}=0.65\right)$.

\section{Central nervous system recurrence}

BL recurred in 167 patients (26\%), with 39 (6\%) presenting a CNS recurrence (21 with and 18 without CNS involvement at diagnosis) (Online Supplementary Figure S2A).
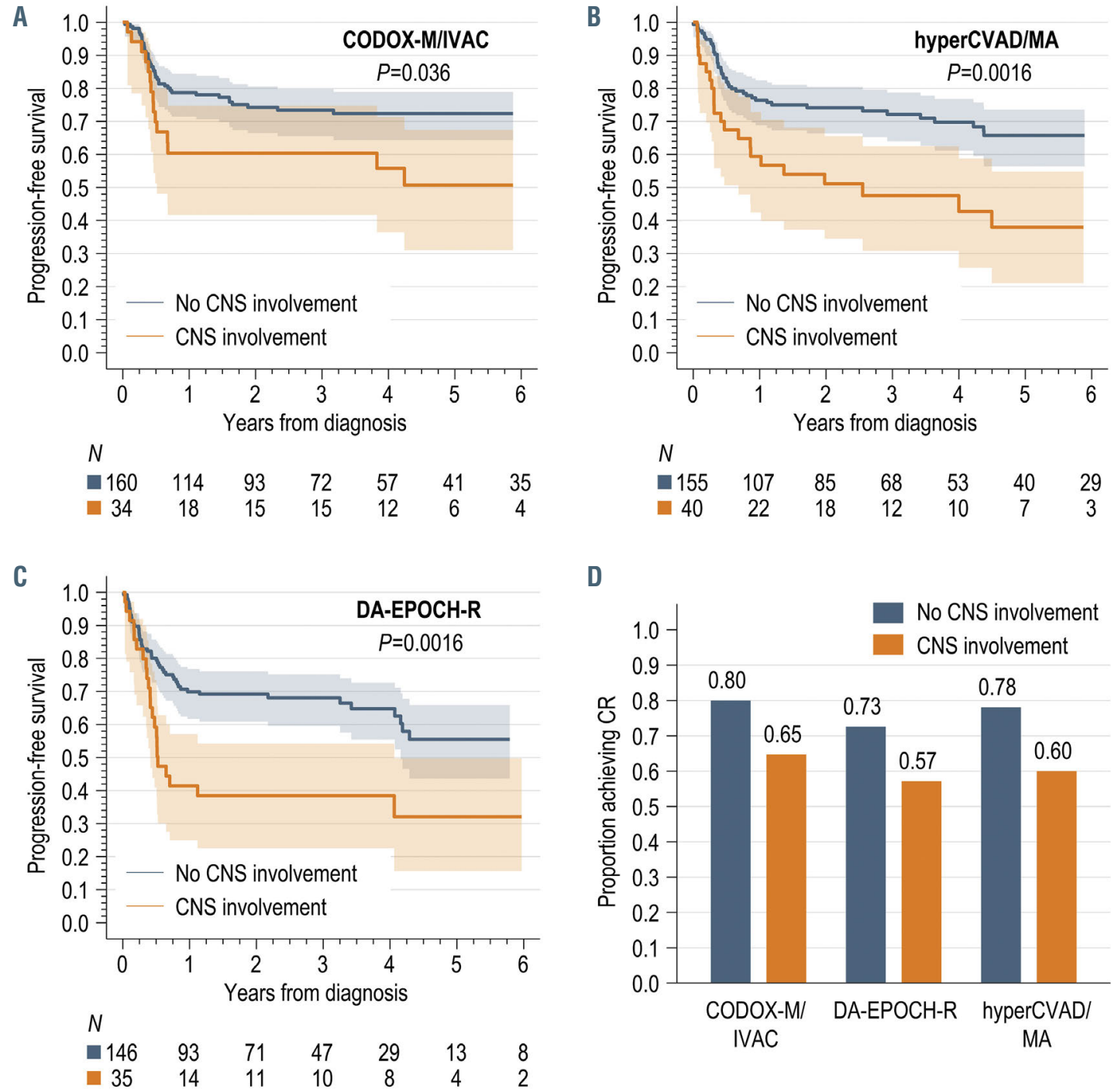

D

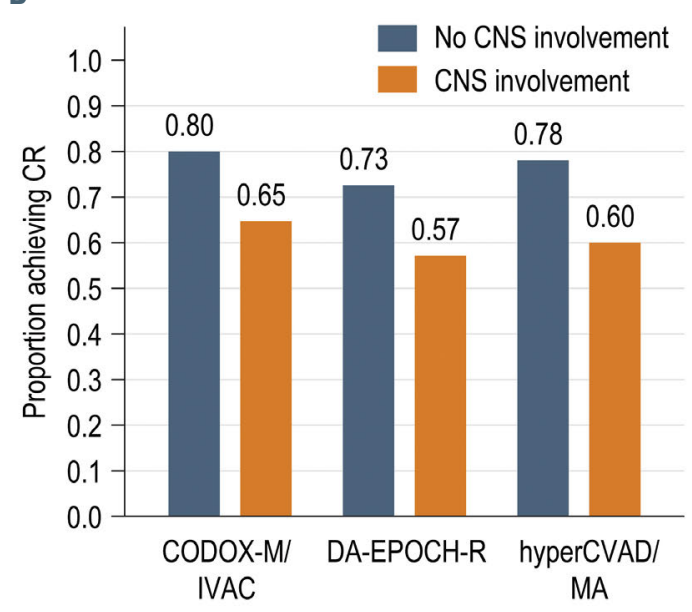

Figure 2. Prognostic significance of baseline central nervous system involvement among patients treated with specific first-line chemotherapy regimens. (A-C) Progression-free survival stratified by the presence of central nervous system (CNS) involvement at diagnosis, for patients treated with: (A) CODOX-M/IVAC ( $\mathrm{n}=194$ ); (B) hyper-CVAD/MA ( $n=195)$; and (C) DA-EPOCH-R $(n=181)$. Shaded areas indicate $95 \%$ confidence interval bands; 3-year survival estimates and $P$-values from a logrank test are listed; the summary $P$-value for interaction between baseline CNS involvement and chemotherapy regimen was 0.85 . (D) Proportions of patients achieving complete response (CR) to first-line therapy, stratified by specific regimen and baseline CNS involvement; the summary $P$-value for interaction between CNS involvement and chemotherapy regimen was 0.95 . 
The median time from diagnosis to CNS recurrence was 5.8 months (IOR, 4.3-7.6). Thirty-four CNS recurrences (87\%) occurred during the first year of follow-up and $32(82 \%)$ involved the CNS alone, without systemic disease. Thirtyseven (95\%) patients with CNS recurrence had stage 4 disease at initial diagnosis, and the only one with early-stage $\mathrm{BL}$ had CNS progression during treatment with $\mathrm{RCHOP}$ (rituximab, cyclophosphamide, doxorubicin, vincristine, and prednisone). The location of the CNS recurrence was only the leptomeninges in $52 \%$ of cases, only parenchyma in $22 \%$, both compartments in $19 \%$, and unspecified in $7 \%$.

Prior to CNS recurrence, $81 \%$ of patients had received rituximab, $97 \%$ had received intrathecal chemotherapy, and $44 \%$ had received HDMTX. Of note, 22 patients (56\%) did not attain a complete response before progression in the $\mathrm{CNS}$, and 18 of those had residual/recurrent disease limited only to the CNS. Following the CNS recurrence, $77 \%$ of patients received salvage treatment: systemic chemotherapy $(64 \%)$, intrathecal chemotherapy $(8 \%)$, or radiation $(5 \%)$. Only five $(13 \%)$ achieved a second complete response and the median survival after CNS recurrence was 2.8 months (range, 2.0-4.2) (Online Supplementary Figure S2B). All five survivors received HDMTX-based salvage therapy. Four of them had received first-line DA-EPOCH-R, one underwent consolidative autologous stem cell transplantation, and one received an allogeneic stem cell transplant.

Analysis of the cumulative incidence of CNS recurrence was conducted in the subcohort of 570 patients who received one of the $\mathrm{NCCN}$-recommended first-line regimens: CODOX-M/IVAC, hyperCVAD/MA, or DA$\mathrm{EPOCH}-\mathrm{R}$. The cumulative incidence of $\mathrm{CNS}$ recurrence in this subcohort was 6\% [4-8] at 1 year (Figure 3A). In univariate analysis, the strongest associations we observed were between CNS recurrence and stage 4 disease (Figure 3B), baseline CNS involvement (Figure 3C), and testicular involvement, this last being very rare (Table 2). Other significant factors included HIV infection, poor PS, $\geq 2$ extranodal sites, LDH level $>3 \times$ XULN, and bone marrow involvement (Online Supplementary Figure S3). In a two-variable model, stage 4 disease (adjusted SHR=7.68 [1.01-58.40], $P=0.049$ ) and baseline CNS involvement (adjusted SHR $=4.04$ [2.08-7.87], $P<0.001$ ) were cumulatively associated with CNS recurrence. Due to the limited number of events, we did not explore more complex multivariate models. Achievement of complete response after first-line therapy was associated with a lower risk of CNS recurrence (SHR=0.30 [0.15-0.57], $P<0.001$ ), whereas receipt of rituximab was not (SHR=1.51 [0.36-6.39], $P=0.58)$.

\section{Central nervous system recurrence according to first-line treatment regimen}

We examined the risk of CNS recurrence according to first-line treatment regimen, comparing regimens that contain high-dose, CNS-penetrant systemic agents (CODOXM/IVAC or hyperCVAD/MA; n=389) with DA-EPOCH-R $(\mathrm{n}=181)$. There was no significant difference between these two groups in the prevalence of baseline CNS involvement $(P=0.93)$, stage 4 disease $(P=0.79), \mathrm{LDH}$ level $>3 \times \mathrm{xULN}$ $(P=0.31), \geq 2$ extranodal sites $(P=0.27)$, or testicular involvement $(P=0.20)$. However, patients selected for DAEPOCH-R were older (median age 54 vs. 44 years; $P<0.001)$, and more likely to have poor PS $(30 \%$ vs. $18 \%$; $P=0.002)$, HIV infection (33\% vs. $20 \%$; $P<0.001)$, or low
A

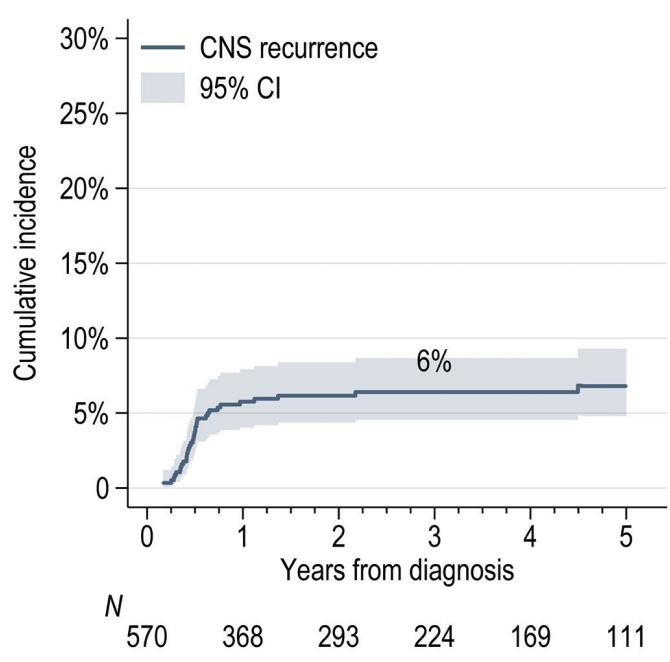

B

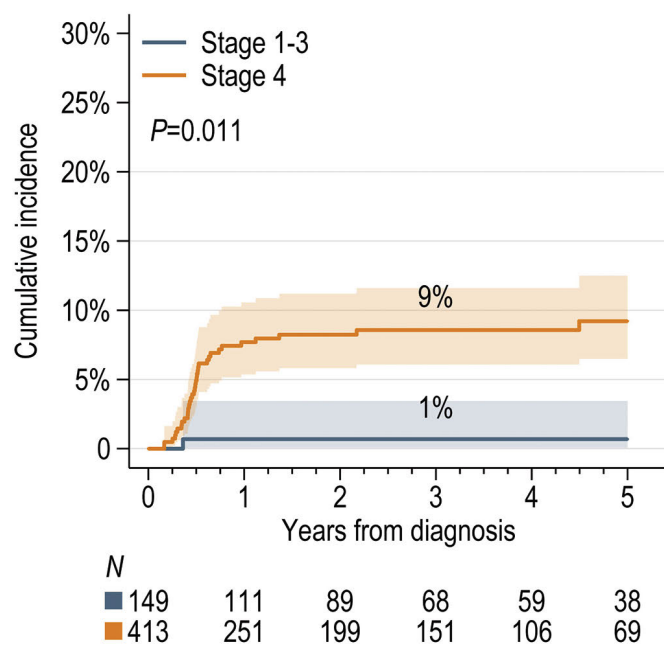

C

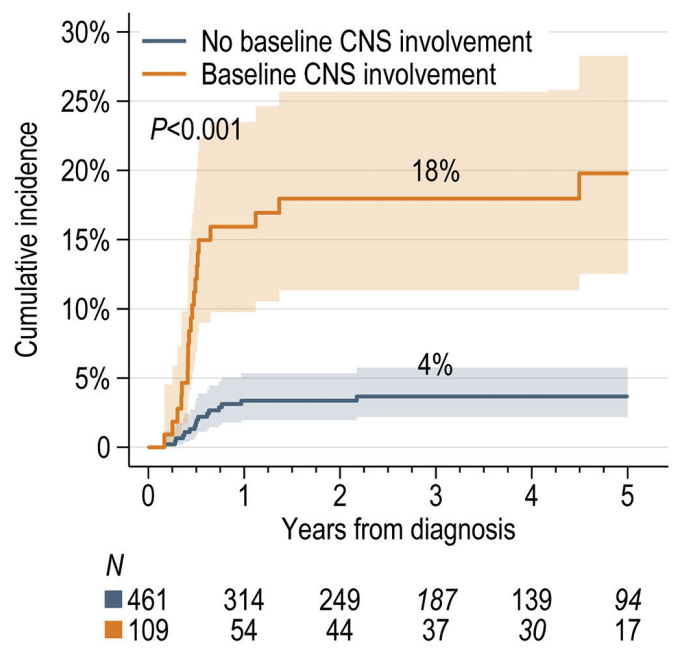

Figure 3. Cumulative incidence of central nervous system recurrence. (A-C) The cumulative incidence of central nervous system (CNS) recurrence in: (A) all patients $(n=570)$ who received chemotherapy with CODOX-M/IVAC, hyperCVAD/MA, or DA-EPOCH-R; (B) stratified by disease stage; and (C) stratified by the presence of baseline CNS involvement at diagnosis. Shaded areas indicate $95 \%$ confidence interval bands; $P$ values are from univariate competing risk models; estimates at 3 years are presented. 
Table 2. Risk factors for central nervous system recurrence in Burkitt lymphoma.

\begin{tabular}{|c|c|c|c|c|c|c|c|}
\hline \multirow{3}{*}{ Variable } & \multicolumn{4}{|c|}{ Cumulative incidence at 3 years } & \multicolumn{3}{|c|}{ Univariate model } \\
\hline & \multicolumn{2}{|c|}{ With } & \multicolumn{2}{|c|}{ Without } & \multirow[t]{2}{*}{ SHR } & \multirow[t]{2}{*}{$95 \%$ Cl } & \multirow[t]{2}{*}{$P$} \\
\hline & $\%$ & $95 \%$ Cl & $\%$ & $95 \%$ Cl & & & \\
\hline Age $\geq 40$ years & 6 & $(4-9)$ & 7 & $(4-11)$ & 0.92 & $(0.47-1.79)$ & 0.80 \\
\hline Age $\geq 60$ years & 5 & $(2-11)$ & 7 & $(5-9)$ & 0.86 & $(0.38-1.95)$ & 0.72 \\
\hline Female sex & 9 & $(5-14)$ & 6 & $(4-8)$ & 1.57 & $(0.78-3.13)$ & 0.20 \\
\hline HIV infection & 11 & $(6-17)$ & 5 & $(3-8)$ & 2.04 & $(1.05-4.00)$ & 0.036 \\
\hline Stage 4 & 9 & $(6-12)$ & 1 & $(0-3)$ & 13.47 & $(1.83-98.9)$ & 0.011 \\
\hline B symptoms & 7 & $(4-11)$ & 6 & $(4-9)$ & 1.25 & $(0.65-2.40)$ & 0.50 \\
\hline ECOG PS 2-4 & 11 & $(6-17)$ & 5 & $(3-7)$ & 2.31 & $(1.14-4.67)$ & 0.019 \\
\hline Hemoglobin $<11.5 \mathrm{~g} / \mathrm{dL}$ & 9 & $(6-14)$ & 4 & $(2-7)$ & 2.54 & $(1.26-5.11)$ & 0.009 \\
\hline Albumin $<3.5 \mathrm{~g} / \mathrm{dL}$ & 9 & $(5-13)$ & 5 & $(3-8)$ & 1.84 & $(0.92-3.66)$ & 0.08 \\
\hline $\mathrm{LDH}>\mathrm{ULN}$ & 7 & $(5-10)$ & 6 & $(2-11)$ & 1.48 & $(0.62-3.55)$ & 0.38 \\
\hline LDH >3x ULN & 10 & $(6-14)$ & 4 & $(3-7)$ & 2.30 & $(1.17-4.50)$ & 0.016 \\
\hline $\mathrm{LDH}>5 \mathrm{x}$ ULN & 10 & $(6-16)$ & 5 & $(3-8)$ & 2.04 & $(1.05-3.97)$ & 0.036 \\
\hline$\geq 2$ extranodal sites & 9 & $(6-14)$ & 4 & $(2-7)$ & 2.13 & $(1.09-4.16)$ & 0.027 \\
\hline $\begin{array}{l}\text { Involvement at diagnosis: } \\
\text { CNS }\end{array}$ & 18 & $(11-26)$ & 4 & $(2-6)$ & 573 & (2. 98-110) & $<0.001$ \\
\hline Bone marrow & 9 & $(6-14)$ & 5 & $(3-7)$ & 2.14 & (1.09-4.17) & 0.026 \\
\hline Intestine & 5 & $(2-11)$ & 7 & $(5-9)$ & 0.74 & $(0.29-1.88)$ & 0.52 \\
\hline Liver & 8 & $(3-16)$ & 6 & $(4-9)$ & 1.30 & $(0.54-3.14)$ & 0.55 \\
\hline Pancreas & 9 & $(2-24)$ & 6 & $(4-9)$ & 1.31 & $(0.32-5.30)$ & 0.70 \\
\hline Pleura/peritoneum & 5 & $(2-11)$ & 7 & $(5-9)$ & 0.76 & $(0.27-2.15)$ & 0.60 \\
\hline Kidney/adrenal & 4 & $(1-12)$ & 7 & $(5-9)$ & 0.61 & $(0.15-2.58)$ & 0.51 \\
\hline Testis $^{\mathrm{a}}$ & 26 & $(6-52)$ & 5 & $(3-8)$ & 5.93 & $(1.74-20.2)$ & 0.004 \\
\hline Uterus/ovary ${ }^{a}$ & 8 & $(1-31)$ & 9 & $(5-15)$ & 0.97 & $(0.13-7.35)$ & 0.97 \\
\hline Female breast $^{\mathrm{a}}$ & 8 & $(1-29)$ & 9 & $(5-15)$ & 0.90 & (0.11-7.22) & 0.92 \\
\hline
\end{tabular}

${ }^{a}$ Men or women only, as pertinent. $95 \%$ CI: $95 \%$ confidence interval; CNS: central nervous system;ECOG PS: Eastern Cooperative Oncology Group performance status; HIV: human immunodeficiency virus; LDH: lactate dehydrogenase; SHR: subhazard ratio; ULN: upper limit of normal.

albumin concentration (48\% vs. $34 \% ; P<0.001)$ (Online Supplementary Table S2).

The 3-year risk of CNS recurrence was significantly lower after CODOX-M/IVAC $(4 \%$ [2-8]) or hyperCVAD/MA (3\% [1-6]) than after DA-EPOCH-R (13\% [8-18]; SHR=3.57 [1.83-6.97]; $P<0.001$ ) (Figure 4A and B). Furthermore, recurrences involved the CNS more frequently after DA-EPOCH-R (40\%) than after the other two regimens $(16 \%, P<0.001)$. The risk was higher regardless of baseline CNS involvement $\left(P_{\text {mexacion }}=0.99\right)$ (Figure $4 \mathrm{C}$ and D),

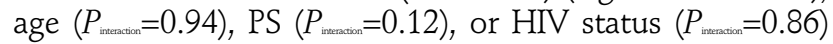
(Online Supplementary Figure S4A-F). Among patients with baseline CNS involvement receiving DA-EPOCH-R, the 3year incidence of CNS recurrence reached 35\% [20-51\%]. The risk did not differ significantly between patients treated with CODOX-M/IVAC or hyperCVAD/MA within any subset. The increased risk of CNS recurrence after DAEPOCH-R persisted adjusting for age, PS, stage 4 disease, HIV positivity, baseline CNS involvement, and testicular involvement (adjusted SHR=4.38 [2.16-8.87], $P<0.001$ ).

All patients with CNS recurrence after DA-EPOCH-R treatment had received intrathecal chemotherapy during their initial therapy with a median of six (IOR, 5-12) doses of methotrexate and a median of four (IOR, 0-5) doses of cytarabine, but strict adherence to the protocol-defined schedule was reported in only $57 \%$ of this subgroup. CNS recurrence after DA-EPOCH-R treatment was leptomeningeal in $63 \%$, intraparenchymal in $25 \%$, and in both compartments in $12 \%$ of cases. Seven patients ( 6 with baseline leptomeningeal disease) received prophylactic HDMTX ( \pm cytarabine) upon completion of DA-EPOCH-R, but $\mathrm{BL}$ recurred in five, including three with a CNS recurrence. Among all patients receiving DA-EPOCH-R, factors significantly associated with $\mathrm{CNS}$ recurrence included baseline CNS involvement ( $\mathrm{SHR}=5.97$ [2.59-13.79]; $P<0.001$ ), marrow involvement (SHR=2.57 [1.07-6.14]; $P=0.034)$, LDH level $>3 x U L N(S H R=2.53$ [1.08-5.94]; $P=0.033)$, and $\geq 2$ extranodal sites ( $\mathrm{SHR}=3.29 \quad[1.28-8.44] ; \quad P=0.013)$. However, adjusting for baseline CNS involvement, no other variable retained statistical significance.

\section{Dlscussion}

In this large, multi-institutional dataset of patients with $\mathrm{BL}$ treated with contemporary immunochemotherapy regimens, CNS involvement at diagnosis was an independent prognostic factor for PFS and OS, and a strong predictor of subsequent CNS recurrence. CNS recurrences developed early and almost exclusively in stage $4 \mathrm{BL}$. No patient with stage 1 or 2 disease experienced a CNS recurrence after treatment with NCCN-recommended regimens. The risk of CNS recurrence was significantly lower with regimens containing high-dose systemic CNS-penetrating agents. However, we observed that among patients with baseline CNS involvement who were treated with DA-EPOCH-R, intrathecal therapy was frequently (in 55\%) not administered according to the schedule in the published protocol, which may have contributed to the high observed incidence of CNS recurrence (35\%). Our results have important implications for the management of $\mathrm{BL}$ in clinical practice, given the rarity of the disease and the paucity of randomized trials. 
A
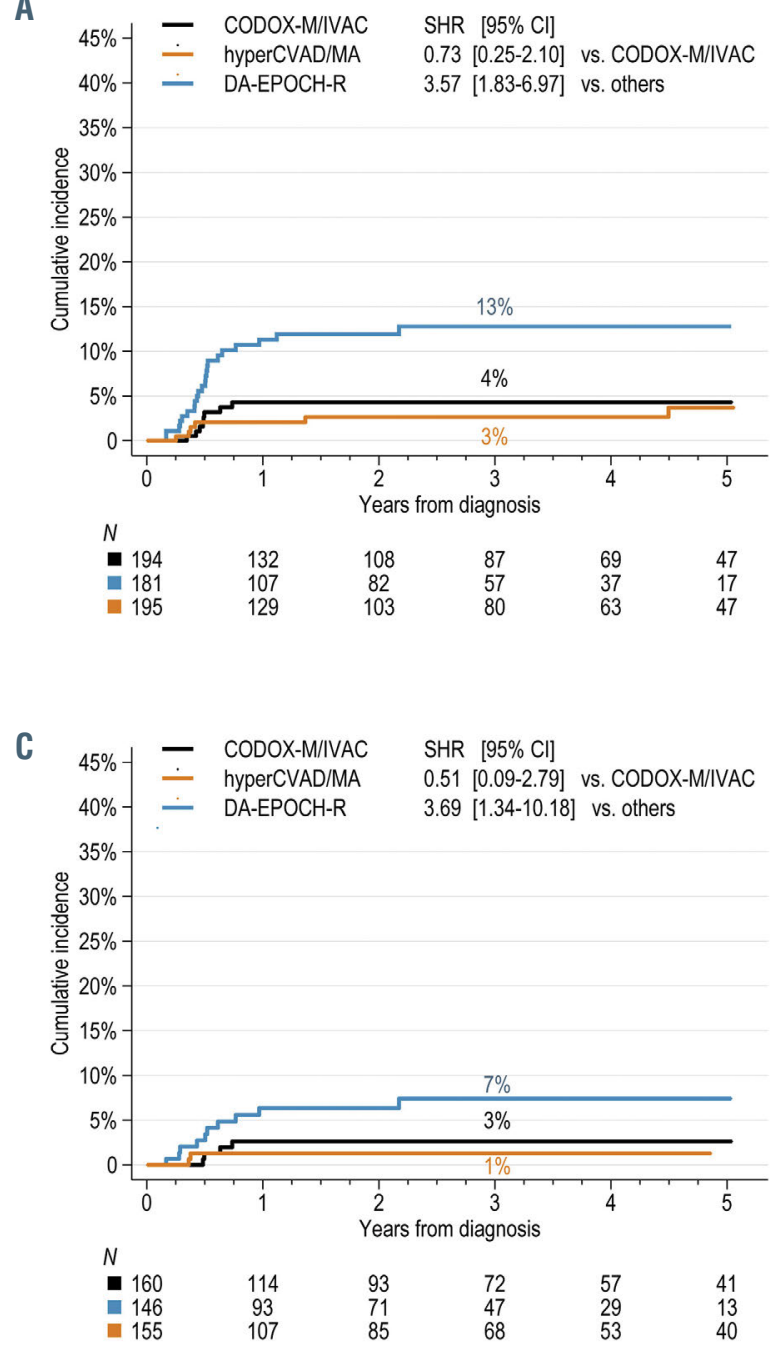

B

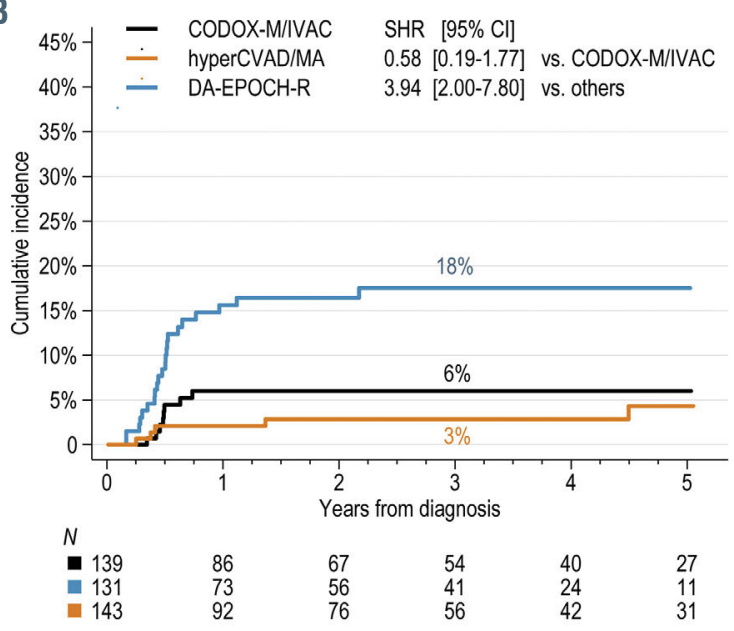

D

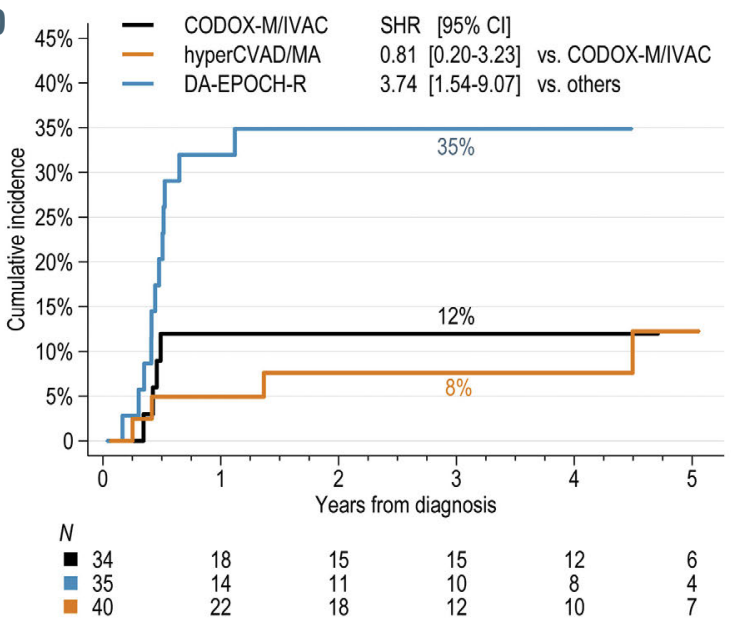

Figure 4. Cumulative incidence of central nervous system recurrence according to first-line chemotherapy regimen. (A-D) The cumulative incidence of central nervous system (CNS) recurrence in: $(A)$ all patients $(n=570)$ treated with CODOX-M/IVAC, hyper-CVAD/MA, or DA-EPOCH-R; (B) patients with stage 4 disease $(n=413)$; $(C)$ patients without CNS involvement at diagnosis; and (D) patients with CNS involvement at diagnosis. Subhazard ratios were derived from univariate competing-risk models comparing hyperCVAD versus CODOX-M/IVAC, or DA-EPOCH-R versus both high-intensity regimens combined. $95 \%$ Cl: $95 \%$ confidence interval; SHR: subjazard ratios.

The prevalence of baseline CNS involvement in this real-world BL cohort (19\%) is higher than that in phase II trials $(10-14 \%)^{8,9,11-13,21-24}$ or in a recent retrospective study of 264 patients treated with immunochemotherapy $(8 \%),{ }^{5}$ but lower than that in a phase III trial $(25 \%) .{ }^{10}$ These differences may reflect both selection bias in smaller trials, and less rigorous CNS staging in routine practice (compared with the phase III setting), in which the use of CSF cytology or flow cytometry is not standardized. Our study had sufficient power to demonstrate independent impacts of CNS involvement on rates of complete response, PFS, and OS in $\mathrm{BL}$, as suggested by some trials, $8,13,22,25$ but not by others., ${ }_{9,10,21,24,26}$

Interestingly, the choice of using an immunochemotherapy regimen did not differ according to CNS involvement, and CNS involvement was prognostically unfavorable regardless of the use of first-line rituximab or any specific regimen, including those that contained HDMTX. Collecting granular data on CNS-directed intrathecal therapy proved challenging in this retrospective study, but intensive administration schedules designed for patients with CNS involvement appeared difficult to execute in real-world practice. All regimens used in our cohort (hyperCVAD/MA, CODOX-M/IVAC, and DA-EPOCH$\mathrm{R}$ ) involve intensified intrathecal regimens for patients with CNS disease, which requires thorough staging to identify subclinical CNS invasion and expertise in the delivery of intrathecal agents, including the use of intraventricular devices. We could not determine whether these schedules were correctly applied in most patients, and whether barriers to effective CNS-directed therapy were related to physicians' preference, patients' refusal, or system-level factors. Furthermore, we did not have data on the use of intraventricular reservoirs, although their availability may affect the efficacy of intrathecal therapy. Both poor prognosis with CNS involvement and suboptimal delivery of CNS-directed therapy indicate that patients with CNS disease need more efficacious and practicable treatment approaches that can be consistently administered in routine clinical practice.

CNS recurrence after standard immunochemotherapy regimens was uncommon (6\%) and exclusive to patients 
with stage 4 disease. Of note, over half of CNS recurrences emerged in patients who did not attain complete response, suggesting primary refractory disease or inadequate control of baseline CNS involvement. Clinical trials have reported lower incidences (0-4\%) of CNS recurrence, , $, 1,1,1,2,1,2,2,27,28^{2}$ which may reflect selective enrollment of lower-risk patients in trials, or suboptimal CNS-directed therapy in our real-world sample. Despite high rates of intrathecal therapy, one in five patients with baseline CNS involvement succumbed to a CNS recurrence, which was predominantly leptomeningeal. Survival after CNS recurrence was dismal and consistent with generally poor outcomes of recurrent BL. ${ }^{729.31}$ HDMTX salvaged a few patients, but mainly those who did not receive it as part of their initial treatment regimen.

An emerging finding was the lower risk of CNS recurrence among patients treated with regimens that contain high-dose systemic CNS-penetrant agents compared with DA-EPOCH-R, which relies solely on intrathecal chemotherapy for CNS management. Our observation could not be explained by stage distribution or prevalence of baseline CNS involvement, which were similar among patients receiving these strategies. Although patients selected for DA-EPOCH-R were older and had a worse PS, possibly compromising the intensity of treatment and adherence to CNS prophylaxis, the increased risk persisted after adjustment for these factors. In this context, it is important to note that we could not ascertain specific CNS staging procedures performed in our cohort. Patients enrolled in the clinical trials of DA-EPOCH-R uniformly underwent CSF evaluations using flow cytometry. ${ }^{12,13}$ Underdiagnosis of occult leptomeningeal disease might result in suboptimal intrathecal treatment, as DA-EPOCH$\mathrm{R}$ relies on an intensive intrathecal regimen (starting with twice-weekly administration) in cases with CSF involvement, whether detected by cytology or flow cytometry. The NCI-9177 study enrolled no patients with parenchymal CNS disease, and out of 11 subjects with CSF involvement, six $(55 \%)$ experienced relapse or death. ${ }^{12,13}$ Considering this experience and the outcomes observed in our series, DA-EPOCH-R should likely be avoided for treatment of BL with parenchymal CNS involvement. Among 81 high-risk patients without baseline CSF involvement, two developed a parenchymal CNS recurrence despite prophylaxis with eight intrathecal injections. In contrast, we observed CNS recurrence in $18 \%$ of patients with stage $4 \mathrm{BL}$ receiving DA-EPOCH-R and in 35\% among those with baseline CNS involvement. We could not discern the reasons for the lower intensity of the intrathecal administration schedule during DA-EPOCH-R (median 6 doses), which possibly contributed to the high failure rate of CNS control; adherence was poor even in academic centers. Because of unavoidable immortal-time bias, we could not compare outcomes based on degree of adherence in our retrospective data. It is possible that with stricter adherence the difference in CNS recurrence between patients treated with the different regimens would not have been significant. However, $37 \%$ of recurrences involved the parenchymal CNS compartment, which may not be prevented with intrathecal therapy. On the other hand, reassuringly, there were no instances of CNS recurrence in patients with disease stages 1-3, supporting the observation of $100 \%$ PFS in low-risk BL after three cycles of DA-EPOCH-R without any CNS prophylaxis. ${ }^{13}$ Because most patients in our study received intrathecal chemotherapy, we could not identify a subgroup that could omit it. We highlight an unmet need for prospective studies of augmentation of DA-EPOCH-R for patients with CNS involvement using a CNS-directed strategy that is more efficacious and feasible to execute in routine clinical practice. The few attempts at "consolidative" HDMTX ( \pm cytarabine) after DA-EPOCH-R observed in our sample do not enable any true interpretation of treatment effect, as these patients had CNS involvement and were likely at particularly high perceived risk of recurrence. The unfavorable outcomes of this approach may reflect the delayed HDMTX administration, lack of added benefit beyond that of intensive intrathecal therapy in leptomeningeal disease, or simply a matter of bias. The need to adjust doses based on degree of cytopenias, early emergence of CNS recurrences, and high failure rate of "consolidative" HDMTX prophylaxis in our admittedly small sample illustrate the challenges of designing such an augmentation. ${ }^{32,33}$ We point out that an analysis of this dataset, as well as other retrospective studies, does not suggest significant differences in the overall PFS or OS according to the first-line treatment regimen used in $\mathrm{BL}^{4,5,16}$

The strengths of this study include its multicenter scope encompassing academic and community-based practices, HIV-associated BL, and a large subset with CNS involvement to allow for an in-depth analysis. Limitations include the retrospective design, rarity of CNS recurrence, and variation in CNS-directed staging or treatment, particularly limited information on the use of CSF flow cytometry, intensity of intrathecal regimens and mode of delivery (intraventricular catheter or lumbar puncture). We also lacked molecular data that could provide insights into the biology of CNS invasion and inform future treatment strategies.

In conclusion, despite the success of immunochemotherapy in BL, patients with CNS involvement constitute a high-risk group in need of better management. In this large real-world dataset, CNS involvement was associated with worse prognosis regardless of first-line regimen applied, and independently of other factors. Regimens incorporating high-dose systemic CNS-penetrant agents were associated with a lower risk of CNS recurrence when administered to patients with baseline CNS involvement in routine clinical practice. Selective use of DA-EPOCH-R for patients with advanced age and worse PS, who could not maintain the intrathecal administration schedule inherent to this protocol, may have influenced our findings. An important insight from our analysis is that when clinicians apply the DA-EPOCH-R regimen, they should strictly follow the pre-specified CNS staging procedures (including CSF flow cytometry) and adequately tailored intrathecal administration schedules, as frequent deviations from the protocol may lead to suboptimal CNS control. CNS-related outcomes from the ongoing HOVON-127BL randomized trial comparing DA-EPOCH-R with CODOX-M/IVAC (EudaraCT 2013-004394-27) will be critical to further define optimal therapy, provided that sufficient numbers of patients with baseline CNS involvement are enrolled. Further prospective studies are needed to optimize realistic delivery of CNS-directed prophylaxis with all standard regimens and to mitigate the incidence of CNS recurrence.

\section{Disclosures}

$A E$ has received research funding from Takeda and Merck; has received honoraria from Research to Practice; has received honoraria from and provided consultancy services for Seattle Genetics, 
Verastem, Affimed and Bayer; and has received honoraria from and sat on a DMC for Pharmacyclics. AD has received research funding from Aptose Biosciences, Gilead Sciences, Takeda Oncology and Bristol-Myers Squibb; has received research funding and provided consultancy services for AstraZeneca, Genentech, Bayer Oncology and Verastem Oncology; has acted as a consultant for Beigene. TG Therapeutics, Celgene, Nurix and Rigel Pharmaceuticals; and is a Leukemia and Lymphoma Society Scholar in Clinical Research. SDS has received research funding from Incyte Corporation, Seattle Genetics, Portola Pharmaceuticals, Pharmacyclics, Acerta Pharma BV, Genentech and Denovo Biopharma; he has received research funding from and acted as a consultant for Merck Sharp \& Dohme Corp; and he has been a member of Astra Zeneca's Board of Directors or advisory committees and has received research funding from this company; a Astra Zeneca. An immediate family member has received research funding from Ignyta, Bristol-Myers Squibb and Ayala. SN has provided advisory board services for Celgene and Sanofi. NR has acted as a consultant for KITE Pharma, Abbvie and Celgene; has received research funding from Genentech; and has provided consultancy services for and received research funding from BMS. UF has received honoraria from Celgene and research funding from Kite Pharma. NE has received honoraria from Pharmacyclics and taken part in a speakers bureau for Verastem Oncology. NK has been a member of the Board of Directors or an advisory committee of Seattle Genetics and Abbvie; has received research funds from Bristol Myers; and has delivered educational content or symposia for Janssen. JPA has received honoraria from Targeted Oncology; and acted as a consultant for OncLive. An immediate family member has had contacts with Puma Biotechnology, Agios, Inovio Pharmaceuticals and Foundation Medicine. MSY has received honoraria from Bayer; has received research funding from Genentech; and has acted as a consultant for Octapharma and Abbvie. CD has received research funding from Denovo, Incyte, LAM Therapeutics, MEI, Millenium/Takeda and Trillium; and has acted as consultant for and received research funding from Bristol-Myers Squibb, Genentech, Merck and Seattle Genetics. RK has sat on speakers' bureaus for Takeda, AstraZeneca and BeiGene; has supplied advisory board services for Karyopharm; and has sat on speakers' bureaus and advisory boards and received research support from Kite, Gilead, BMS, Celgene and Juno. PM has acted as a consultant for Celgene, Teneobio, Karyopharm, Janssen, Sandoz and I-MAB. GM has participated in speakers' bureaus for Tevan Oncology. MK has acted as a consultant for Pharmacyclics, AstraZeneca and Celgene; has sat on speakers' bureaus for Seattle Genetics; and is an employee of the University of Colorado. ML has acted as a consultant for Spectrum, Seattle Genetics, Portola, OncLive, Novartis. Kite, Gilead Sciences, Inc., DAVA, Bayer, AbbVie, VANIUM and Verastem; has received research funding from MiRagen and Curis; and has acted as a consultant for and received research funding from TG Therapeutics, Juno Therapeutics and Janssen Scientific Affairs, LLC. CP has received research funding from Xencor, Roche/Genentech, Infinity, TG Therapeutics, AbbVie and Acerta/AstraZeneca; has acted as a consultant for Pharmacyclics, Janssen, Amgen and Bayer, and has provide consultancy services for and received research funding from Genentech, BeiGene and Kite. ISL has been a member of the Board of Directors or an advisory committee of Janssen Scientific and Seattle Genetics; and has received research funding from the NIH. AJO has received research funding from Genentech, Adaptive Biotechnologies, TG Therapeutics and Spectrum Pharmaceuticals. The remaining authors report no significant conflicts of interest.

\section{Contributions}

$A S Z, A M E, I S L$ and AJO designed the research, performed the analysis, and wrote the manuscript draft. ASZ and $A M E$ contributed equally. $A D, S D S, D J, L A L, C W, S-H K, S N, S S u$, $N R, U F, V P K, N E, K A B, N K, D S, J P A, A G, S Y, C D, E R, G V$, $R K, Y S, A T, M B, P M, S S t, A C, Y K C, A S-E, A K, M C C$, $K M B, S B, B M H, V M O-N, C D^{\prime} A, D A B, S M M, R V, G M$, AS, MJG, KAD, MS, PC, MK, MAL, NP, PV, CAP, VB and $T P$ contributed patients' data, and edited and approved the manuscript.

\section{Funding}

This work was conducted with voluntary support of the contributing institutions and researchers, without any external funding.

\section{References}

1.Zayac AS, Olszewski AJ. Burkitt lymphoma: bridging the gap between advances in molecular biology and therapy. Leuk Lymphoma. 2020;61(8):1784-1796.

2. Sariban E, Edwards B, Janus C, Magrath I. Central nervous system involvement in American Burkitt's lymphoma. I Clin Oncol. 1983;1(11):677-681.

3. Boehme V, Zeynalova S, Kloess M, et al. Incidence and risk factors of central nervous system recurrence in aggressive lymphoma--a survey of 1693 patients treated in protocols of the German High-Grade NonHodgkin's Lymphoma Study Group (DSHNHL). Ann Oncol. 2007;18(1):149 157

4. Oosten LEM, Chamuleau MED, Thielen FW, et al. Treatment of sporadic Burkitt lymphoma in adults, a retrospective comparison of four treatment regimens. Ann Hematol. 2018;97(2):255-266.

5. Jakobsen LH, Ellin F, Smeland KB, et al Minimal relapse risk and early normalization of survival for patients with Burkitt lymphoma treated with intensive immunochemotherapy: an international study of 264 real-world patients. $\mathrm{Br}$ J
Haematol. 2020;189(4):661-671

6. Thomas DA, Cortes J, O'Brien S, et al. Hyper-CVAD program in Burkitt's-type adult acute lymphoblastic leukemia. J Clin Oncol. 1999;17(8):2461-2470.

7. Woessmann W, Zimmermann M, Meinhardt A, et al. Progressive or relapsed Burkitt lymphoma or leukemia in children and adolescents after BFM-type first-line therapy. Blood. 2020;135(14):1124-1132.

8. Thomas DA, Faderl $S$, O'Brien $S$, et al. Chemoimmunotherapy with hyper-CVAD plus rituximab for the treatment of adult Burkitt and Burkitt-type lymphoma or acute lymphoblastic leukemia. Cancer. 2006;106(7):1569-1580.

9. Rizzieri DA, Johnson JL, Byrd JC, et al. Improved efficacy using rituximab and brief duration, high intensity chemotherapy with filgrastim support for Burkitt or aggressive lymphomas: cancer and Leukemia Group B study 10 002. Br J Haematol. 2014;165(1):102-111.

10. Ribrag V, Koscielny S, Bosq J, et al. Rituximab and dose-dense chemotherapy for adults with Burkitt's lymphoma: a randomised, controlled, open-label, phase 3 trial. Lancet. 2016;387(10036):2402-2411

11. Lacasce A, Howard O, Lib S, et al. Modified magrath regimens for adults with Burkitt and Burkitt-like lymphomas: preserved efficacy with decreased toxicity. Leuk Lymphoma. 2004;45(4):761-767.

12. Dunleavy K, Pittaluga S, Shovlin $M$, et al. Low-intensity therapy in adults with Burkitt's lymphoma. N Engl J Med. 2013;369(20):1915-1925.

13. Roschewski M, Dunleavy K, Abramson JS, et al. Multicenter study of risk-adapted therapy with dose-adjusted EPOCH-R in adults with untreated Burkitt lymphoma. J Clin Oncol. 2020;38(22):2519-2529.

14. Alderuccio JP, Lossos IS. DA-EPOCH-R for adult Burkitt's lymphoma: pros and cons. J Oncol Pract. 2018;14(11):676-678.

15. NCCN Clinical Practice Guidelines in Oncology (NCCN Guidelines®): B-cell lymphomas. Version 4.2020 - August 13, 2020. Available from: https://www.nccn.org/professionals/physician_gls/pdf/b-cell.pdf.

16. Evens AM, Danilov AV, Jagadeesh D, et al. Burkitt lymphoma in the modern era: real world outcomes and prognostication across 30 US cancer centers. Blood. 2021;137 (3):374-386.

17. Swerdlow SH, Campo E, Pileri SA, et al. The 2016 revision of the World Health 
Organization classification of lymphoid neoplasms. Blood. 2016;127(20):2375-2390.

18. Cheson BD, Pfistner B, Juweid ME, et al. Revised response criteria for malignant lymphoma. J Clin Oncol. 2007;25(5):579586.

19. Fine JP, Gray RJ. A proportional hazards model for the subdistribution of a competing risk. J Am Stat Assoc. 1999;94(446):496509.

20. White IR, Royston P, Wood AM. Multiple imputation using chained equations: issues and guidance for practice. Stat Med. 2011;30(4):377-399.

21. Noy A, Lee JY, Cesarman E, et al. AMC 048: modified CODOX-M/IVAC-rituximab is safe and effective for HIV-associated Burkitt lymphoma. Blood. 2015;126(2):160-166.

22. Hoelzer D, Walewski J, Dohner $\mathrm{H}$, et al. Improved outcome of adult Burkitt lymphoma/leukemia with rituximab and chemotherapy: report of a large prospective multicenter trial. Blood. 2014;124(26):38703879 .

23. Corazzelli G, Frigeri F, Russo F, et al. RDCODOX-M/IVAC with rituximab and intrathecal liposomal cytarabine in adult Burkitt lymphoma and 'unclassifiable' highly aggressive B-cell lymphoma. Br J Haematol. 2012;156(2):234-244.

24. Mead GM, Barrans SL, Oian W, et al. A prospective clinicopathologic study of dose-modified CODOX-M/IVAC in patients with sporadic Burkitt lymphoma defined using cytogenetic and immunophenotypic criteria (MRC/NCRI LY10 trial). Blood. 2008;112(6):2248-2260.

25. Wasterlid T, Brown PN, Hagberg O, et al. Impact of chemotherapy regimen and rituximab in adult Burkitt lymphoma: a retrospective population-based study from the Nordic Lymphoma Group. Ann Oncol. 2013;24(7):1879-1886.

26. Mead GM, Sydes MR, Walewski J, et al. An international evaluation of CODOX-M and CODOX-M alternating with IVAC in adult Burkitt's lymphoma: results of United Kingdom Lymphoma Group LY06 study. Ann Oncol. 2002;13(8):1264-1274.

27. Divine M, Casassus P, Koscielny S, et al. Burkitt lymphoma in adults: a prospective study of 72 patients treated with an adapted pediatric LMB protocol. Ann Oncol. 2005;16(12):1928-1935

28. Patte C, Auperin A, Michon J, et al. The Societe Francaise d'Oncologie Pediatrique LMB89 protocol: highly effective multia- gent chemotherapy tailored to the tumor burden and initial response in 561 unselected children with B-cell lymphomas and L3 leukemia. Blood. 2001;97(11):3370-3379.

29. Short NJ, Kantarjian HM, Ko H, et al Outcomes of adults with relapsed or refractory Burkitt and high-grade B-cell leukemia/lymphoma. Am J Hematol 2017;92(6):E114-E117.

30. Decker DP, Egan PC, Zayac AS, Treaba DO, Olszewski AJ. Treatment strategies and risk of central nervous system recurrence in high-grade B-cell and Burkitt lymphoma. Leuk Lymphoma. 2020;61(1):198201.

31 Maramattom LV, Hari PN, Burns LJ, et al. Autologous and allogeneic transplantation for burkitt lymphoma outcomes and changes in utilization: a report from the center for international blood and marrow transplant research. Biol Blood Marrow Transplant. 2013;19(2):173-179.

32. Gastwirt JP, Roschewski M. Management of adults with Burkitt lymphoma. Clin Adv Hematol Oncol. 2018;16(12):812-822.

33. Dunleavy K. Approach to the diagnosis and treatment of adult Burkitt's lymphoma. Oncol Pract. 2018;14(11):665-671. 